Part 1

\section{Speculative Trading: Physicists' Forays into Finance}

\section{Toby Chapman}

The raising of interest rates on the Hong Kong stock exchange last October led to a short financial crisis on markets in the West. The turmoil had its origins in worries over Thailand's economy - subsequent devaluations of currencies in the Far East left investors focusing on Hong Kong, and to stem the attack on their currency the Hong Kong authorities raised interest rates, as they always do. This caused a crash on the Honk Kong stock market as prices fell. And as a shock wave spread across the time zones, markets in the West lost value also.

But a financial crisis in the West doesn't need bad news from the Far East to trigger it, a fact which has become known to physicists who study stock exchanges. According to a recent computer model, the details of which were published in Europhysics Letters last December, the actions of speculative traders alone is enough to bring about a crisis.

The model, built by University of Fribourg physicist Yi-Cheng Zhang and two co-workers, Guido Caldarelli and Matteo Marsili, is a simplified version of the traders world: a stock market with only one stock, with no interest rates and, most importantly, stripped of all external influences (bad news from the Far East, for example).

This virtual trading floor is populated by traders doing only one thing: trading to make as much money as possible. In each buying round they speculate on the future price of the one stock, buying and selling according to their own strategy, which is based on the recent price history. In effect, each trader has a method for predicting the future price based on recent price changes. The price itself is then determined by the division of demand by supply. Two twists are that traders can learn from each other's strategies, and the worst trader in each round is thrown off the trading floor and replaced.

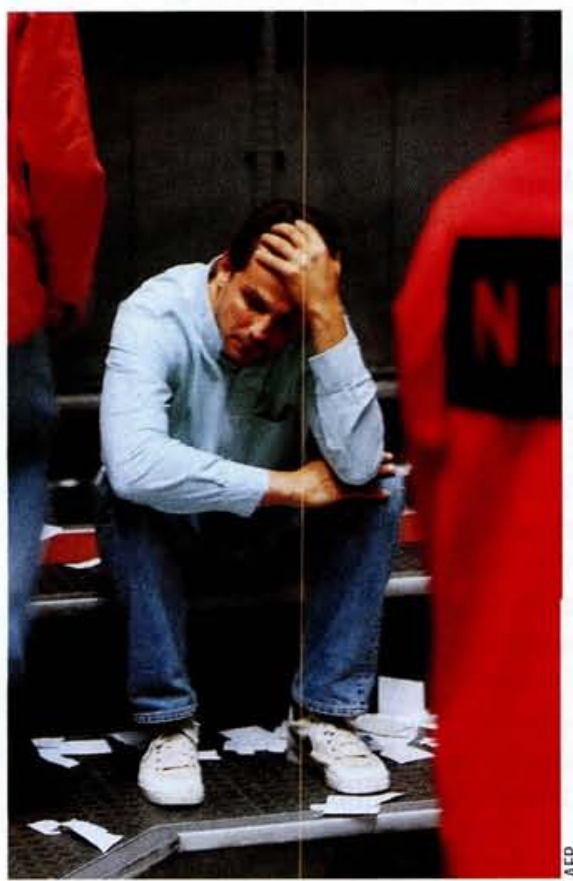

Don't be too depressed, you only have yourself and your fellow traders to blame. An options trader on the palais Brongniart trading floor in Paris, at the end of a day on which the French stock exchange fell $4.27 \%$. This day, 28th October 1997, was near the end of last year's turmoil, caused by crises in the Far East-the kind of crisis that can result from the actions of traders alone

As the buying rounds progress, price fluctuations emerge that are remarkably similar to those of a real stock exchange. And this similarity to a real market is despite the fact that only simple speculative trading takes place, and the outside world has no influence - something that might surprise real traders who rely heavily on news from the economic world outside their trading floor.

But in Zhang's model, the financial world is reduced to trading only. 'Traders only have to trade to be better than their fellow traders by buying and selling at the right moment. If you do so on average better than your fellow traders, you win. Despite such simplicity we have a model that gives a price history which is very similar to real market data,' he says.

Zhang compares the fractal dimensions of his data with an analysis of a real stock market (in Nature 376 p46). But one of his co-workers draws attention away from this: 'I do not attach much significance to the fact that we obtained very good numerical values. It is not the main point,' says Marsili. 'The understanding that we got is that the statistics of price histories in financial markets can be understood as the result of the internal interaction, and not the fundamental interaction with the external world.'

Zhang's model best simulates foreign exchange markets, as most transactions there are speculative. And it does produce the occasional crash. Which suggests that in the real world speculative trading is the dominant cause of instability, and not bad news from Hong Kong.

This model is only one of a family of market models created in recent years by physicists. And the field itself, called econophysics by practitioners, is peppered both with high regard and slight disagreement. Didier Sornette, an econophysicist at Nice University, is cautious about comparing a crash in Zhang's model with a real crisis: 'I agree with YiCheng Zhang by saying that crashes are probably intrinsic to the system, but they are not obtained by his model' - real world crashes are far more severe.

So will the model be of use to traders? Market traders don't really care about this kind of theoretical model, Zhang warns. And Marsili points out that it doesn't tell you whether to buy or to sell tomorrow, it is a tool for understanding rather than a tool for prediction.

Zhang should know how distant his model is from the methods of the trading floor: 'I personally traded for two months, trading on the foreign currency market in London. Every minute the price changes. It's very, very stressful. You receive orders, in five seconds you have to judge whether to take the offer or leave it. You see the price, then you make your computer model, then you sell or you buy.'

Zhang's work is also a long way from that of most economic theoreticians, he points out. They don't have the physicist's desire for simplification. 'It is not really in their taste to promote such a brutal mode, without even interest rates. It is not in their culture. Maybe we don't have such a prejudice, because we are physicists, and we do not have to make a living in their field.' See One Hundred Years of Physics in Finance, page 35. 